\title{
Decision Tree Applied to Learning Relations between Ontologies
}

\author{
R. Mirambicka, A. Razia Sulthana, and G. Vadivu
}

\begin{abstract}
An ontology is a structural framework for organizing information in a domain. There may be different ontologies describing the same domain. This introduces heterogeneity and difficulty in integrating information. Thus arises the need for finding relations between ontologies. Decision tree classifier is a robust machine learning tool that can be used to learn relations between two ontologies constructed in the same domain and find links between any pair of classes taken from the ontologies.
\end{abstract}

Index Terms-Decision tree, ontology, ontology relations, machine learning.

\section{INTRODUCTION}

The term 'ontology' means different in different fields. In philosophy 'ontology' refers to the theory of nature of existence and in artificial intelligence, an ontology gives a formal representation of a domain, a vocabulary of terms and the relations between the terms. The major reason for constructing ontologies is to share and reuse domain knowledge among researchers and enable machine to machine communication [1]. For example, if a group of researchers develop a detailed ontology describing an e-commerce website, then others intending to start an e-business can simply use the information provided in the ontology instead of starting from scratch.

Just as there may be differences of opinion and perceptions among people concerning a matter of interest, different ontologies can be constructed for the same domain of interest There is a pressing need to integrate and create a common link between them [2]. To make the fact more concrete, take for example, the management committees of two different universities. They may create their own version of a university ontology. An 'Assistant Professor' in one university may be referred to as a 'Junior Lecturer' in another University. A person who worked as an Assistant Professor in the first University may shift to a Junior Lecturer position in the second University. If the two University ontologies had been linked, then there would be no problem in finding the current job position and location of the person.

Decision tree classifiers are intuitive and easy to assimilate by humans. Their construction does not require any domain knowledge and is therefore appropriate for exploratory

Manuscript received January 24, 2013; revised April 10, 2013.

R. Mirambicka and A. Razia Sulthana are with the Department of Information Technology, SRM University, Kattankulathur-603203, TamilNadu, India (e-mail: mirasri9@gmail.com, itrazia@gmail.com).

G. Vadivu is with the Department of Information Technology, SRM University, Kattankulathur- 603203 (e-mail: vadivu.g@ktr.srmuniv.ac.in). knowledge discovery [3]. Decision trees help model data in a tree-like or graph-like structure. They are constructed with the help of a training data matrix which is derived from the input ontologies. Attribute selection measures like Gini index, Information Gain are used to select the attribute that best partitions the matrix rows into distinct classes. Decision trees are constructed in a top-down recursive divide- and-conquer manner, following a hill-climbing greedy approach. The model is then tested using unknown pairs, a path is traced from the root node to the leaf node, and the relation between the two classes each taken from different ontologies, is determined. Many of the branches may reflect noise on the training data matrix [3] various tree pruning attempts help to improve the classification accuracy on the data. Efficiency becomes a cause of concern as the training set may grow too large. Suggestions are given in Section IV-Precautionary Measures.

The major focus of the technique described in the subsequent sections, is on the superclass of relation. [2] describes many efforts to find equivalence relations between pairs of classes each taken from two different ontologies, but the superclass of relation is equally important and the deduction that whether a class from the first ontology is a superclass of a class taken from the second ontology and vice versa, can actually strengthen and 'enhance' the discovery of equivalence classes between the two ontologies. Again, a point to note, the technique so described, enhances the discovery of unknown useful relations between two classes taken from two different ontologies, describing the same domain of interest and is not a full-fledged alignment or merging technique.

A perceived advantage of this method is its non-dependence on external resources. The method fully exploits the class pairs from both ontologies while generating the training data matrix. This is in contrast to [4] where there is a detailed description of many similarity based methods which depend on WordNet. There are different strategies for ontology matching like string similarity, synonyms, structure similarity and based on instances. WordNet can help improve such similarity measures. It divides the terms into three databases and groups terms into 'Synsets'. The ontology matching method can refer to these groups of terms and recognizes equivalent terms while matching pair of concepts between ontologies.

One can perceive a close structural similarity between a decision tree and the tree-like representation of an ontology. The approach can be described in another way as integration of both the ontologies and formation of a new tree- the decision tree, which provides a new perspective to the structure of the two ontologies and thus becomes a useful tool 
to find out the link between the two ontologies.

\section{PROBlem Definition}

An ontology $\mathrm{O}=(\mathrm{S}, \mathrm{A})$ is a pair where $\mathrm{S}$ is the ontological signature containing all the terms of the specific domain and $\mathrm{A}$ is the set of axioms which clearly specifies the meaning of the terms in the signature [5]. Various relations like subclassOf, superclassOf, equivalence are all set of axioms and are included in A. The ontologies consist of classes, properties, individuals and annotations. The collection of all these terms is the signature. For example, referring to Fig. 1, of GovernmentX ontology, the classes are GovernmentX, Executive, President, Judiciary and so on. The properties are consists of, is Head of and so on. Properties relate the individuals or instances of one class to another class and they also serve as attributes to the class. Without properties an ontology just becomes a word list with no relevance and meaning. Annotations further clarify the purpose and meaning of classes. They include comments, labels and definitions of terms.

The construction of ontologies requires deep knowledge of the domain of interest. The ontologies consist of several terms arranged hierarchically and having explicit and implicit relationships between them. Finding correspondences between two ontologies in the same domain is where the challenge lies. In [6], W3C-World Wide Web Consortium has recommended Extensible Mark up Language XML, XML-Schema, Resource Description Framework (RDF), RDF-Schema, and Web Ontology Language (OWL). OWL Language is a by-product of the evolution of these languages and shares the advantages of both XML and RDF. It adds more vocabulary by giving more description to classes, properties, their relationships and so on.

Consider two ontologies $\mathrm{O}_{1}=\left(\mathrm{S}_{1}, \mathrm{~A}_{1}\right)$ and $\mathrm{O}_{2}=\left(\mathrm{S}_{2}, \mathrm{~A}_{2}\right)$ which describe the same domain and need to be aligned. The objective is to set a relation between a pair of classes (Class1, Class2) where Class1-left-side class is a class in ontology $\mathrm{O}_{1}$ and Class2-right-side class is from ontology $\mathrm{O}_{2}$. This relation can be, for example, equivalence or superclass of relation [6]. Once the relation between the pair of classes is established, it will act as an enhancement tool in the process of alignment or merging of the two ontologies.

The objective of the method proposed is to use the decision tree classifier to deduce whether a pair of classes (Class1, Class2) are related via the superclass of relation i.e. whether Class 1 of Ontology $\mathrm{O}_{1}$ is a superclass of Class 2 in Ontology $\mathrm{O}_{2}$. The training data matrix consists of rows whose labels have been already deduced; 'Yes' if Class1 is a superclass of Class2 and 'No' otherwise. Only class pairs which have explicit or implicit property specifications are taken into consideration for this exercise.

\section{STEPWISE SOLUTION}

The stepwise solution consists of 4 phases: Exploitation phase, Construction of training data matrix, Construction of the decision tree classifier and the testing phase. This is explained diagrammatically in Fig. 3. Notice in Fig. 3 about the arrows which move from ontologies to the training and testing matrices. The two arrows which point to the training data matrix are separate, which means that the class pairs are taken from separate ontologies. The two arrows are joined together while pointing to the testing data matrix because the left-side class is taken from first ontology and the right-side class is taken from the second ontology.

\section{A. Exploitation Phase}

Two ontologies preferably in the OWL-DL language [6] and in the same domain which has been completely synchronized with reasoning tools are considered. For example, Fig. 1 and Fig. 2 depict ontologies in the same domain which are the structures of GovernmentX and GovernmentY. Between each pair of classes there must be some defined relations or inferred relations.

Ontology 0

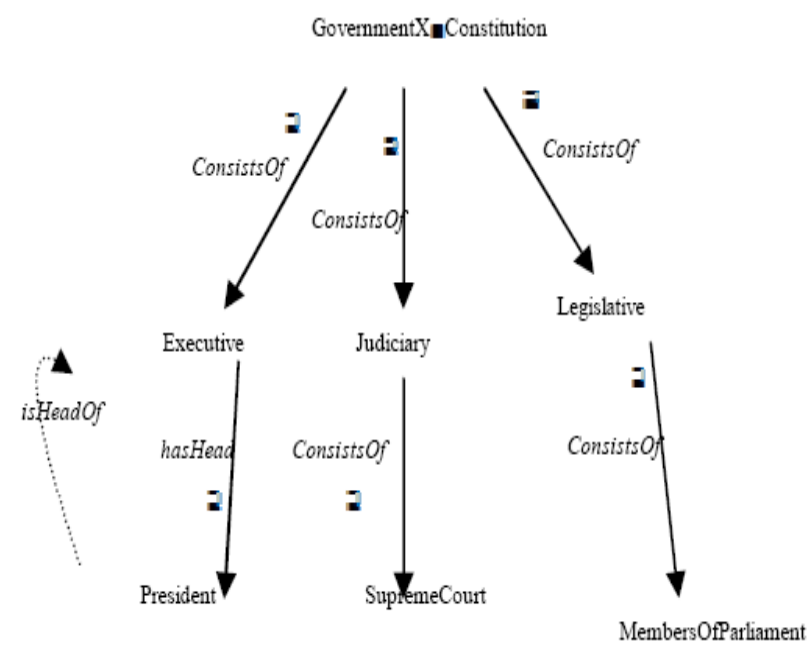

Fig. 1. Ontology of GovernmentX

Ontology $\mathrm{O}_{2}$

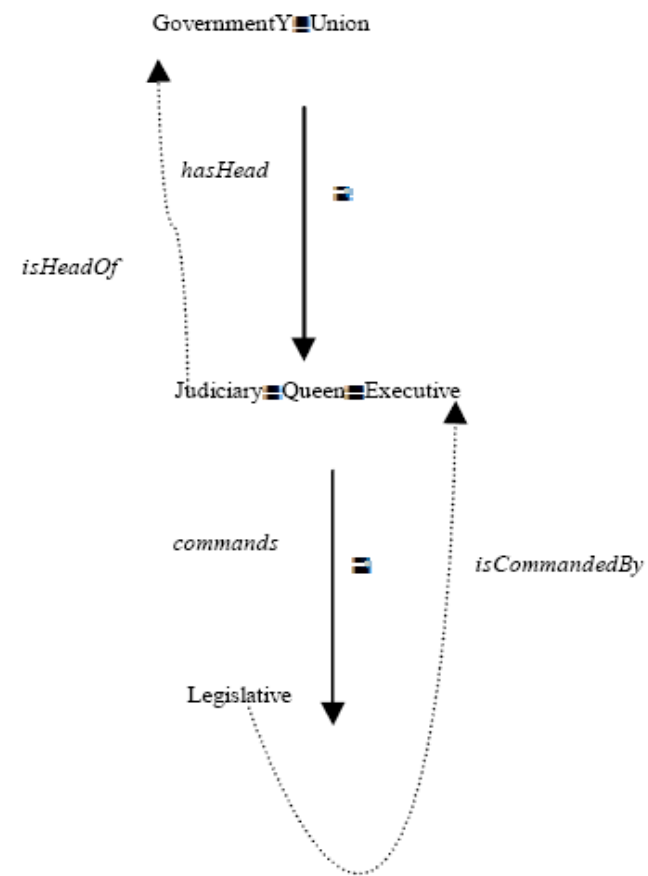

Fig. 2. Ontology of GovernmentY. 
TABLE I: TRAINING DATASET MATRIX

\begin{tabular}{|c|c|c|c|c|c|c|}
\hline $\begin{array}{l}\text { Properties } \\
\text { Class Pairs } \\
\end{array}$ & " hasHead & isHeadOf & "Commands & isCommandedBy & "ConsistsOf & Label \\
\hline (GovernmentX, Executive) & Right & Right & None & None & Both & Yes \\
\hline (GovernmentY, Judiciary) & Both & Both & Right & Right & None & Yes \\
\hline (Executive, Judiciary) & Left & Left & None & None & None & No \\
\hline
\end{tabular}

Fig. 1 for example, depicts the ontology of GovernmentX with classes like Executive and Judiciary. Arrow tail denotes the domain and Arrow head -range of the properties (like consists of, has Head) marked in italics. The symbol $\supseteq$ denotes superclassOf derived from common sense reasoning tools and $\equiv$ denotes equivalence. For example, GovernmentX is equivalent to Constitution in Fig. 1. As another example in Fig. 2, GovernmentY is a superclass of Judiciary which is again a superclass of Legislative. Thus by using reasoning tools it can be inferred that Government $Y$ is a superclass of Legislative. The properties clearly describe how the classes are related to each other. For example, in Fig. 1 , Consists of property helps to intuitively recognize that the pair of classes are related by a superclass of relation. While exploiting each of the ontologies, it is sufficient to consider only the properties and classes because if an axiom is true for a class, it is true for all the individuals in the class. Since properties relate each pair of classes in a more or less unique manner they are used as columns for the training data matrix.

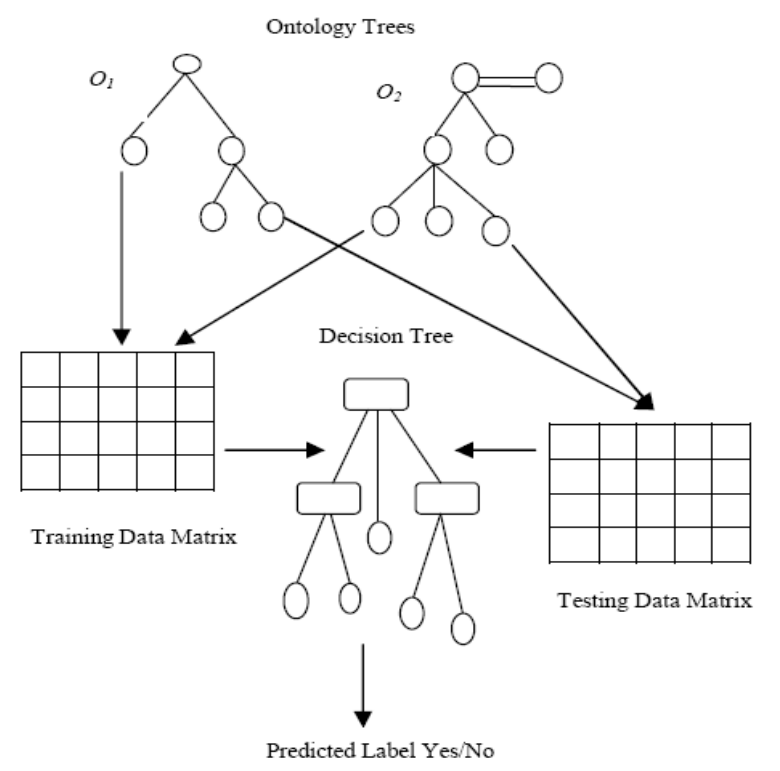

Fig. 3. Stepwise solution diagram.

\section{B. Training Data Matrix}

Classes from each of the ontologies are taken pair wise where both left-side class and right-side class belong to the same Ontology. In Table I, the columns are the properties listed from both the ontologies. If a property is not associated with any class then mark 'None', only left-side class mark 'Left', only right-side class mark 'Right' and 'Both' if association of the property is for both classes. Label column has two values, 'Yes' if the left-side class is a superclass of the right-side pair and 'No' otherwise.

This is the most significant phase in the method. A good choice of the training data matrix is where there is a balance between the 'Yes' and 'No' rows. A lot also depends on the way the ontology has been constructed and how far the relations between two classes in the same ontology have been exploited by reasoning tools.

\section{Construction of the Decision Tree Classifier}

Iterative Dichotomiser- ID3-algorithm is used in the model, which is by far the most basic and oldest decision tree algorithm. The primary advantage of using the ID3 algorithm is that it is robust to noisy and missing data. During phase BConstruction of the Training Data Matrix, there are a lot of chances for missing information especially if the ontologies have a lot of abstract classes. The choice of the input training data matrix makes a lot of difference to testing efficiency of the model. The matrix should have equal number of rows marked 'Yes' and equal no of rows marked 'No'. There is always a bias towards more rows marked 'No', because majority of the classes will be linked via other relations like equivalence, mismatch or overlapping. Thus the method will be more effective if the ontology trees have a lot of hierarchy levels as it would lead to a better choice of the training data matrix.

Decision trees sort out data in a top-down approach from the root to the leaf nodes, where the leaf nodes marked by the rounded symbol in Fig. 3, denote the classification labels 'Yes' or 'No'. The rounded rectangles in Fig. 3 denote the property columns like consists of, is Head of etc, and the edges denote the possible values of the properties like 'None', 'Left', 'Right', 'Both' and so on. The process is repetitive and iterative, dividing the training data matrix into sub-matrices and goes on till no more rows are available for classification or all the rows in the sub-training matrix is of the same classification label. ID3 uses all the training matrix rows at each stage to generate the decision tree, and thus the model is less sensitive to errors and noise in the training data matrix.

Using the training data matrix as in Table I, each property is tested for information gain. The property column with the highest information gain is the splitting property. The construction of the decision tree classifier allows the formation of decision rules. The rules are formulated from root node to the leaf node. For example, referring to Fig. 1. there can be a rule like If consists of property is marked 'Left' and has Head property is marked 'Right' then Label is 'Yes'. Thus, the decision tree modeling helps in gaining a new perspective to the ontology trees and also helps in finding out new relationships between the terms of the respective ontologies. The ID3 algorithm and methods to prune the tree are explained clearly in [3], [7].

Other decision tree algorithms like C4.5 and CART mentioned in [3], [7], can also be used to construct the classification model. The algorithmic steps are the same as ID3 but the Attribute Selection Measures are different. C4.5 
uses Gain Ratio and CART uses a measure called Gini Index. These alternate measures can be used for a comparative study and the final model can be an average of all the three models.

\section{Testing Phase}

The testing data matrix is similar to Table I. The only difference is that the left- side class must belong to Ontology $O_{1}$ and the right-side class must belong to Ontology $O_{2}$. For example (GovernmentX, GovernmentY) could be a testing class pair. Using the decision tree model, it is possible to predict the relation between two classes. Since the decision tree classifier was constructed by exploiting classes from the same ontologies, the model is applied to predict the relation between a class from ontology 1 and a class from ontology 2 . This aids in finding correspondences between the two ontologies and enhances the possible merging of the two ontologies constructed in the same domain.

If say, for example from the decision tree it is analysed that President in $\mathrm{O}_{1}$ is a superclass of Queen in $\mathrm{O}_{2}$ and Queen in $\mathrm{O}_{2}$ is also a superclass of President in $\mathrm{O}_{1}$, then President and Queen are equivalent classes. Suppose for both (Class1, Class2) and (Class2, Class1) testing pairs the predicted label is 'No', then there is no clear prediction between the relation of Class 1 and Class2. They could be equivalent or disjoint. Again suppose for (Class1, Class2), the label is 'Yes' and (Class2, Class1) the label marked is 'No', then it can be deduced that Class 1 and Class 2 are connected by a pure superclass of relation.

\section{Precautionary Measures}

One of the most important precautionary step to be taken is the choice of the appropriate dataset for the training data matrix. Decision tree classifier is a probabilistic data mining tool. Whether the model is accurate enough is a huge question which needs to be answered. The method depends heavily on the choice of the training dataset matrix described in Table I. Sampling must be done carefully to avoid overfitting or underfitting of data. In an experimental study [3], overfitting reduces the accuracy of the tree by 10 to 25 percent. The input training data matrix must neither have too many rows labeled 'Yes' nor labeled 'No'. Both must be given equal preferences. Abstract classes i.e. classes without properties are ignored.

It is interesting to note that individual pairs are not being considered in this method. It is deemed sufficient that if Class 1 in $\mathrm{O}_{1}$ is a superclass of Class 2 in $\mathrm{O}_{2}$ then all individuals in Class2 belong to Class1.

Also the training data matrix tends to grow very large with the size of ontologies. One way to reduce this is that equivalent properties can be ignored in the column list. In Fig 1 'has Head' property is the inverse of the property 'is Head of'. If the number of properties is too large then one of them, not both can be considered. Nevertheless, it is better to take all the properties into consideration and other methods like SLIQ [8] and SPRINT [9] can handle very large training sets. SLIQ employs a memory-resident entry list and disk resident property column list. Each row in the matrix is identifies by a row-id which links both the entry list and property column list. SPRINT employs different lists for each property column and each row is identified by a row-id. The lists consist of the property column, label column and the row-id. When a split occurs at a tree node, the lists are partitioned and distributed to the child nodes accordingly. The order of the records in the list is maintained and the need for resorting does not arise.

Since the training data matrix grows large, the decision tree also tends to grow very large. Post pruning attempts include converting the tree into decision rules which have been mentioned before and retaining only one of the rules which have an equivalent meaning. In [7] the various advantages of converting the decision tree into rules are described. Rules help improve readability and better interpretation of the data and helps speed up the decision process.

An alternate suggestion is that instead of creating one large tree, a Random forest can be generated which has the advantage of handling very large data and producing accurate results. A Random forest is generated by using a random training row generator and creating different possible trees. The final result can be an aggregate prediction of all the trees generated.

During the testing phase, it is advisable to use class names which have been already used while creating the training data matrix. Since the method is a supervised learning approach, predictions will be more accurate for those pairs already used to train the matrix. For example, if (GovernmentX, Executive) from first Ontology in Fig. 1 and (GovernmentY, Judiciary) from the second Ontology in Fig. 2 are training pairs, then (GovernmentX, Judiciary) and (Executive, GovernmentY) can be used in the generation of the testing matrix. This precautionary measure also avoids rows with missing values in the testing data matrix.

\section{CONCLUSION}

The method described works very well for ontologies in which every pair of classes have some defined relations between them and whose trees have a lot of levels. This can be used independently and does not depend on external tools and resources to find links between pairs of classes.

The discussed method is just another of the many applications of the decision tree classifier. The same training data matrix can also be used against any other classifier like Naïve Bayes, Support Vector Machine and so on for cross verification but as stated before decision trees are the most accurate and intuitive of all the other machine learning techniques. It is robust to errors and can handle very large training data sets with ease.

Another perceived advantage of this method is that each property column has only four possible options, 'None', 'Both', 'Left' and 'Right'. It is mentioned in [7] that the Information gain measure is biased towards columns which have a large number of possible values. Since each column has exactly four possibilities, the bias is avoided and increases the accuracy of the decision tree model.

It is said in the testing phase that if both (Class1, Class2) and (Class2, Class1) testing pairs are marked 'No' then no decision can be taken regarding the testing pairs. The aim of the method described is to find out how many class pairs in 
the testing matrix are related by a superclassOf relation. The same can be applied for other relations and alternative training matrices and corresponding decision trees can be generated. For example, the 'Yes' label could mean disjointness relation and the 'No' label all other relations.

Further ideas for extension include that instead of just a Yes/No prediction numerical values like ' 0 ' for disjoint and ' 1 ' for equivalence and 0.5 for superclassOf/subclassOf will give a clearer perspective of the relations between the left and right side classes.

\section{ACKNOWLEDGMENT}

R. Mirambicka would like to heartily thank her professors and co-authors A. Razia Sulthana, G.Vadivu and Department of Information Technology, SRM University for all the support, encouragement and inputs received for this paper.

\section{REFERENCES}

[1] N. F. Noy and D. L. Mc. Guiness, "Ontology Development 101: A guide to creating your first ontology," Stanford Knowledge Systems Laboratory Technical Report 2001, KSL 01-05, Stanford University.

[2] J. Euzenat and P. Shvaiko, Ontology Matching, NJ: Springer Verlag New York Inc, Secaucus, 2007.

[3] J. W. Han and M. Kamber, Data Mining: Concepts and Techniques, San Francisco: Morgan Kaufmann Publishers Inc., CA, USA, 2000.

[4] F. Lin and K. Sandkuhl, "A Survey of Exploiting WordNet in Ontology Matching," in Proc. IFIP International Federation for Information Processing, Artificial Intelligence and Practice II; Max ramer, Boston: Springer, vol. 276, 2008, pp. 341-350.

[5] V. Spiliopoulos et al., "On the discovery of subsumption relations for the alignment of ontologies," Web Semantics: Sci. Serv. Agents World Wide Web, 2010, doi: 10.1016/j.websem.

[6] OWL Web Ontology Language Review. (February 10, 2004). W3C Recommendation. [Online]. Available: http://www.w3.org/TR/owl-features

[7] T. Mitchell, "Decision Tree Learning," T. Mitchell Machine Learning, The McGraw Hill Companies Inc., 1997, pp. 52-78.

[8] M. Mehta, R. Agrawal, and J. Rissanen, "SLIQ: A Fast Scalable Classifier for Data Mining," Lecture Notes in Computer Science, New York: Springer-Verlag, 1996, pp. 18-32.

[9] J. Shafer, R. Agrawal, and M. Mehta, "SPRINT: A Scalable Parallel Classifier for Data Mining," in Proc. the twenty-Second VLDB Conference, San Francisco, Calif: Morgan Kaufmann, 1996, pp. 544-555.

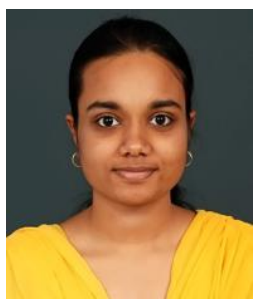

R. Mirambicka completed her Bachelors and Masters degree in Mathematics from Stella Maris College, Chennai, Tamilnadu, India in the year 2011 and is currently pursuing Master of Technology -Database Systems in SRM University- Kattankulathur Campus, Tamilnadu, India. A Gold Medalist, she has received several general proficiency prizes during her academic years in Stella Maris College.

She did a 2 month summer internship project titled 'Fractals, Fractal Interpolation and Iterated Function Systems' at the Indian Institute of Technology, Chennai in the year 2010. She is at present focused on her final year project based on ontology alignment using data mining classification techniques. Keenly interested in both Mathematics and Databases, her current areas of interest include Data Mining, Ontology Matching and Semantic Web.

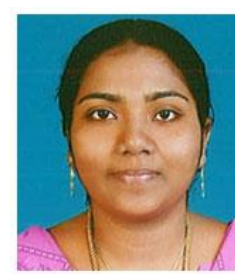

A. Razia Sulthana obtained her Masters in Engineering (M.E) in Computer Science and Engineering from S.A. Engineering College-affiliated to Anna University Chennai, Tamilnadu, India in the year 2011 and Bachelors in Technology (B.Tech) in Information Technology from Jaya Engineering College-affiliated to Anna University, Chennai, Tamilnadu, India in 2007. She is a University first rank holder in M.E. Computer Science.

She has been working as an Assistant Professor-(Ordinary Grade) in SRM University-Kattankulathur Campus, Tamilnadu, India since 2011. She has presented a paper titled “ARIPSO-Association Rule Interactive Post Mining using Schemas and Ontologies" in the IEEE Conference held at St. Xaviers College of Engineering, Kanyakumari, India. Her research interests include Ontologies, Data Warehousing, Cloud Computing and Data Mining.

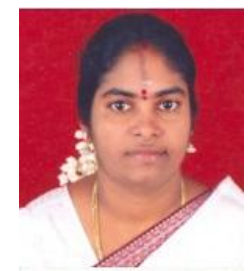

G. Vadivu completed her Masters in Technology, Computer Science and Engineering in the year 2007 and Bachelor of Engineering in Computer Science and Engineering from IRTT, affiliated to Bharathiar University, Tamilnadu, India in the year 1993.

She worked as a lecturer from 2000-2005, then a senior lecturer from 2005-2009 and Assistant

Professor- Senior Grade from 2009 onwards all in the Department of Information Technology, SRM University, Kattankulathur Campus, Tamilnadu, India. She was deputed to Nippon Electric Company(NEC), Japan as part of SRM-NEC collaborative projects. Her research interests include Semantic Web, Data Mining, and Database Systems and she has conducted several workshops on the same.

Ms. Vadivu received the Best Teaching Faculty Award from SRM University for her outstanding support and guidance she has been giving to her students. She also obtained a Certificate of Appreciation for her journal paper titled "Ontology Mapping of Indian Medicinal Plants with standardized Medical Terms," Journal of Computer Science, ISSN 1549-3636, Aug. 2012. 